\title{
Association of miR-27a, miR-181a and miR-570 genetic variants with gallbladder cancer susceptibility on North indian population
}

\author{
Annapurna Gupta1", Kiran L Sharma ${ }^{1}$, Annu Yadav' ${ }^{1}$, Vijay Kumar ${ }^{2}$, Sanjeev Misra ${ }^{2}$, Ashok Kumar ${ }^{3}$, Balraj Mittal ${ }^{1}$ \\ From International Conference on Human Genetics and 39th Annual Meeting of the Indian Society of \\ Human Genetics (ISHG) \\ Ahmadabad, India. 23-25 January 2013
}

\section{Background}

MicroRNAs are small endogenously expressed short non-coding RNAs. They appear to be critical regulators of tumor biology as their aberrant expression is well characterized in cancer progression. The role of microRNA is not fully understood in gallbladder carcinoma, so in present study we investigated the role of miR-27a, miR-181a and miR-570 genetic variants with gallbladder cancer (GBC) susceptibility.

\section{Material and methods}

In this case-control study, we evaluated the role of miR27a, miR-181a and miR-570 genetic polymorphisms with GBC susceptibility in North Indian population. The present study included 515 GBC patients and 200 healthy controls from North India. Genotypes were determined by TaqMan probes. Statistical analysis was done by SPSS ver. 16. In silico analysis was performed using Bioinformatics tools (F-SNP, FAST-SNP).

\section{Results}

Logistic regression analysis showed no significant association of miR-27a, miR-181a and miR-570 genetic polymorphism with GBC susceptibility ( $P>0.05)$. On stratifying our data on the basis of gall stone status, the [AG+GG] genotypes of miRNA rs895819 (A>G) were significantly associated with increased risk of $G B C$ in patients without stone $(\mathrm{p}=0.003 \mathrm{OR}=1.83[(95 \% \mathrm{CI}) 1.23$ 2.72]. The genetic risk by miR-27a, rs895819 (A>G) was also modulated by tobacco consumption as the heterozygotes (AG) were at higher risk $\mathrm{p}=0.005 \mathrm{OR}=1.94$
[(95\%CI) 1.22-3.08]. However, there was no association of miR-181a and miR-570 polymorphisms with disease risk in subgroup analysis. In-silico analysis showed change in transcriptional regulation of miR-27a and miR-570 variations.

\section{Conclusions}

We found significant association of miRNA rs895819 A $>G$ with gallbladder cancer risk through gallstone independent pathway and tobacco usage.

\section{Authors' details}

'Departments of Genetics, Sanjay Gandhi Post Graduate Institute of Medical Sciences (SGPGIMS), Lucknow, India. ²Department of Surgical Oncology, KGMU, Lucknow, India. ${ }^{3}$ Surgical Gastroenterology, SGPGIMS, Lucknow, India.

Published: 21 January 2014

doi:10.1186/1755-8166-7-S1-P17

Cite this article as: Gupta et al:: Association of miR-27a, miR-181a and miR-570 genetic variants with gallbladder cancer susceptibility on North indian population. Molecular Cytogenetics 2014 7(Suppl 1):P17. 